\title{
Geometry of Nonlinear Connections
}

\author{
L. Del Riego ${ }^{1}$ \\ Facultad de Ciencias \\ Zona Universitaria \\ Universidad Autónoma de San Luis Potosí \\ San Luis Potosí, SLP \\ 78290 MEXICO \\ lilia@galia.fc.uaslp.mx
}

\author{
Phillip. E. Parker \\ Mathematics Department \\ Wichita State University \\ Wichita KS 67260-0033 \\ USA \\ phil@math.wichita.edu
}

28 August 2004

\begin{abstract}
We show that locally diffeomorphic exponential maps can be defined for any second-order differential equation, and give a (possibly nonlinear) covariant derivative for any (possibly nonlinear) connection. We introduce vertically homogeneous connections as the natural correspondents of homogeneous second-order differential equations.

We provide significant support for the prospect of studying nonlinear connections via certain, closely associated secondorder differential equations. One of the most important is our generalized Ambrose-Palais-Singer correspondence.
\end{abstract}

MSC(1991): Primary 53C15; Secondary 53C22, 58E10.

${ }^{1}$ Partially supported by CONACYT grant 26594-E. 



\section{Introduction}

Second-order differential equations (SODEs) are an important class of vector fields on the tangent bundle $T M$ of a manifold $M$. So far, only those corresponding to linear connections (classical sprays) are well understood. But nonlinear connections are of real interest, especially in some newer applications $[3,4,5,24,25]$.

In Riemannian geometry, the geodesic spray, whose integral curves are the geodesics of the Levi-Civita connection, has played an important rôle; see, for example, $[9,7]$. In Finsler geometry, four main connections have been used, none of them linear: those of Cartan, Berwald, Hasiguchi, and Chern [6]. Riemannian geometry has been a main thread of mathematics over the last century, and Finsler geometry has recently undergone a great revival. Applications of it now include modeling the singular sets of MongeAmpère equations $[1,16]$, studying the manifold of Hamiltonians $[8,22]$, and modeling river flows and mountain slopes [4].

One of our motivations for this work was the desire to make a comprehensive theory of SODEs and nonlinear connections which would include (pseudo)Riemannian geodesic sprays and analogues for Finsler spaces as examples. We have recovered enough of the (pseudo)Riemannian results to be assured of the correctness of our approach; comparing the results for Finsler spaces will be the subject of future study.

Section 2 contains our notation and conventions. In Section 3 we present the new exponential maps defined by SODEs. Section 4 describes some relations between (possibly nonlinear) connections and SODEs and the associated (possibly nonlinear) covariant derivatives and geodesics. Throughout, all manifolds are smooth (meaning $C^{\infty}$ ), connected, paracompact, and Hausdorff.

The authors thank CONACYT and FAI for travel and support grants, Wichita State University and Universidad Autónoma de San Luis Potosí for hospitality during the progress of the work, and J. Hebda and A. Helfer for helpful conversations. Del Riego also thanks M. Mezzino for writing a Mathematica package for her use.

\section{Review and definitions}

Let $J$ be the canonical involution on $T T M$ and $C$ the Euler (or Liouville) vector field. We recall that in local coordinates, $J(x, y, X, Y)=(x, X, y, Y)$ 
and $C:(x, y) \mapsto(x, y, 0, y)$.

Definition 2.1 A section $S$ of $T T M$ over $T M$ is a SODE when $J S=S$; that is, when it can be expressed locally as $S:(x, y) \mapsto(x, y, y, \mathcal{S}(x, y))$.

Before commenting on this definition, we must briefly digress to consider the notion of homogeneity for functions.

Consider the equation $f(a x)=a^{m} f(x)$. In projective geometry, one requires this to hold only for $a \neq 0$. We call this homogeneous of degree $m$. In other areas, such as Euler's Theorem in analysis, one further restricts to $a>0$. We call this positively homogeneous of degree $m$. Finally, in order that homogeneity of degree 1 coincide with linearity, one must allow any $a \in \mathbb{R}$ (including zero). We call this completely homogeneous of degree $m$ and denote it by $h(m)$.

The difference between homogeneity and complete homogeneity is minor; essentially, it is just the difference between working on $T M-0$ and on $T M$. The difference between positive homogeneity and the other two is more significant. For example, the inward-going and outward-going radial geodesics of the Finsler-Poincaré plane in [6] have different arclengths.

Definition 2.2 We say that a SODE $S$ is homogeneous of degree $m$ when the functions $\mathcal{S}(x, y)$ are completely homogeneous (respectively, homogeneous) of degree $m$ in the vertical component in some induced local coordinates: $\mathcal{S}(x, a y)=a^{m} \mathcal{S}(x, y)$ for some $m \geq 2$ (respectively, $m<2$ ) and all scalars $a \in \mathbb{R}$ (respectively, $a \neq 0$ ).

The break comes at $m=2$ because an $h(m)$ SODE is to be associated with a connection whose homogeneity formula will contain $a^{m-2}$; see (4.3). In the distinguished induced local coordinates, $S:(x, a y) \mapsto\left(x, a y, a y, a^{m} \mathcal{S}(x, y)\right)$. Only induced local coordinates $\left(x^{\prime}, y^{\prime}, X^{\prime}, Y^{\prime}\right)$ related to this $(x, y, X, Y)$ by a block-diagonal transition matrix will preserve the form of such an $S$. Other induced local coordinates preserve the correct degree of homogeneity in the vertical component $Y$, but may change the degree of homogeneity in the "horizontal" component $X$. Thus from now on, we shall use only these admissible atlases on $T M$ when studying homogeneous SODEs et relata; see around (4.1) and after Theorem 4.4. Note that these admissible atlases are simultaneous bundle atlases for both vector bundle structures on TTM.

Remark 2.3 In the extant literature [11, 17, 18, 20, 21], one finds homogeneous vector fields of degree $m$ defined by $[C, S]=(m-1) S$. In any (not just admissible) local coordinates, $S:(x, a y) \mapsto\left(x, a y, a^{m-1} y, a^{m} \mathcal{S}(x, y)\right)$. Thus a homogeneous SODE can be a homogeneous vector field only for $m=2$. 
Hereinafter we shall call $h(2)$ SODEs (quadratic) sprays, in agreement with $[18,20,21]$. (Note that complete homogeneity is required for our quadratic sprays to coincide with the usual spray of [2].) We denote the set of SODEs on $M$ by $\operatorname{QSpray}(M)$ and those which are $h(m)$ by $\operatorname{QSpray}_{m}(M)$. It has been usual to consider only (positive) integral degrees of homogeneity, but we make no such restriction.

We will associate a SODE to each (possibly nonlinear) connection in the rôle of a geodesic spray (see Theorems 4.1 and 4.10), so we shall use the name "quasispray" to reflect this new, extended rôle. We consider only SODEs defined on the entire tangent bundle $T M$; others $[5,6,20]$ have used the reduced tangent bundle with the 0 -section removed, which is appropriate when considering $h(m)$ SODEs when $m<2$ (including $m<0$ ). For $0 \leq$ $m<2$, one usually requires SODEs to be $C^{0}$ across the zero-section; e.g., for Finsler spaces. Most of our results hold mutatis mutandis in these cases as well; any unobvious exceptions are noted specifically.

Several important results concerning (quadratic) sprays $[2,9,15,20]$ rely on the facts that each such spray $S$ determines a unique torsion-free linear connection $\Gamma$, and conversely, every spray $S$ arises from a linear connection $\Gamma$ the torsion of which can be assigned arbitrarily.

In the general case, a (possibly nonlinear) connection $\Gamma$ gives rise to a quasispray $S$ (see Proposition 4.1), but the correspondence has not been well studied before. We shall extend most of the features of the (quadratic) spray-linear connection correspondence to the general setting. One of our ultimate goals is to determine just how well nonlinear connections can be studied via their quasisprays.

Definition 2.4 Let $S$ be a SODE on $M$. We say that a curve $c:(a, b) \rightarrow M$ is a geodesic of $S$ or an $S$-geodesic if and only if the natural lifting $\dot{c}$ of $c$ to $T M$ is an integral curve of $S$.

This means that if $\ddot{c}$ is the natural lifting of $\dot{c}$ to $T T M$, then $\ddot{c}=S(\dot{c})$.

Following this definition, we make a convention: all $S$-geodesics are always to be regarded as extended to the maximal parameter intervals (i.e., to be inextendible) unless specifically noted otherwise. When the SODE $S$ is clear from context, we refer simply to geodesics.

We refer to $[11,13]$ for motivation and further general results, to [11] for results specific to homogeneous SODEs (called homogeneous sprays there), and to [12] for more examples. Note that the SODEs in [11] were positively homogeneous; the extension of those results to complete homogeneity is 
straightforward, once the definition of homogeneous spray there is changed to the one for homogeneous SODE here.

\section{$3 \quad$ Exponential maps}

Let $S$ be a SODE on $M$. We define the generalized exponential maps (plural!) $\exp ^{\varepsilon}$ of $S$ as follows.

First let $p \in M, v \in T_{p} M$, and $c$ be the unique $S$-geodesic such that $\ddot{c}=S(\dot{c}), c(0)=p$, and $\dot{c}(0)=v$. Define

$$
\exp _{p}^{\varepsilon}(v)=c(\varepsilon)
$$

for all $v \in T_{p} M$ for which this makes sense. From the existence of flows (e.g., $[19$, p. 175]), it follows that this is well defined for all $\varepsilon$ in some open interval $\left(-\varepsilon_{p}, \varepsilon_{p}\right)$, which in general depends on $p$, and for all $v$ in some open neighborhood $U_{p}$ of $0 \in T_{p} M$, which in general depends on the choice of $\varepsilon \in\left(-\varepsilon_{p}, \varepsilon_{p}\right)$. This defines $\exp _{p}^{\varepsilon}$ at each $p \in M$.

Next, choose a smooth function $\varepsilon: M \rightarrow \mathbb{R}$ such that $\varepsilon(p) \in\left(-\varepsilon_{p}, \varepsilon_{p}\right)$ for every $p \in M$. (The smoothness of $\varepsilon$ is for our later convenience: we want $\exp _{p}^{\varepsilon}$ to be smooth in $\varepsilon$ as well as in all other parameters.) Then the global map $\exp ^{\varepsilon}$ is defined pointwise by $\left(\exp ^{\varepsilon}\right)_{p}=\exp _{p}^{\varepsilon(p)}$. The domain of $\exp ^{\varepsilon}$ is a tubular neighborhood of the 0 -section in $T M$ and the graph of $\varepsilon$ lies in a tubular neighborhood of the 0 -section in the trivial line bundle $\mathbb{R} \times M$.

Examples show that it is possible that $\varepsilon_{p}<1$ for every open neighborhood of $0 \in T_{p} M$ if the SODE is inhomogeneous; one due to J. Hebda may be found in [13].

The closer the graph of $\varepsilon$ gets to the 0 -section of $\mathbb{R} \times M$, the larger the tubular neighborhood of the 0 -section in $T M$ gets.

Proposition 3.1 For $\varepsilon_{1} \leq \varepsilon_{2}$, we have $\operatorname{dom}\left(\exp ^{\varepsilon_{1}}\right) \supseteq \operatorname{dom}\left(\exp ^{\varepsilon_{2}}\right)$, attaining all of $T M$ for $\varepsilon=0$ when $\exp ^{0}=\pi$.

This puts the bundle projection $T M \rightarrow M$ in the interesting position of being a member of a one-parameter family of maps, all of whose other members are local diffeomorphisms. (This is reminiscent of singular perturbations.)

Theorem 3.2 For every $\varepsilon$ such that $0<|\varepsilon|<\varepsilon_{p}$, the generalized exponential map $\exp _{p}^{\varepsilon}$ is a diffeomorphism of an open neighborhood of $0 \in T_{p} M$ with an open neighborhood of $p \in M$. 
The proof is only a slight generalization of the usual argument as in $[10$, p. 116f]; more details are in [13].

Consider the two-parameter family $\exp _{p}^{\varepsilon}(a v)$. Clearly, $\varepsilon$ is an $S$-geodesic parameter; i. e., the curve obtained by fixing $v$ and varying $\varepsilon$ is an $S$-geodesic through $p$. But, in general, $a$ will not be a geodesic parameter; i.e., the curve obtained by fixing $\varepsilon$ and $v$ and varying $a$ is not an $S$-geodesic through $p$. If $S$ is homogeneous, then $a$ is a geodesic parameter [13]. In any case, the $a$-parameter curves are interesting: they are the integral curves for our new Jacobi vector fields. These were mentioned in [12] and will be studied in more detail elsewhere. Finally, note that we could just as well define exponential-like maps based on the $a$-curves and they would share most of the properties of our new exponential maps.

\section{Connections and their quasisprays}

In general, a connection on a manifold $M$ is a subbundle $\mathscr{H}$ of the second tangent bundle $\pi_{T}: T T M \rightarrow T M$ which is complementary to the vertical bundle $\mathscr{V}=\operatorname{ker}\left(\pi_{*}: T T M \rightarrow T M\right)$, so

$$
T T M=\mathscr{H} \oplus \mathscr{V} .
$$

We note there are two vector bundle structures on $T T M$ over $T M$, denoted here by $\pi_{T}$ and $\pi_{*}$. While $\mathscr{V}$ is always a subbundle with respect to both [23, pp. 18,20], $\mathscr{H}$ is a subbundle with respect to $\pi_{*}$ if and only if the connection is linear [7, p. 32]. Observe that in (any) local coordinates, elements of $\mathscr{V}$ look like $(x, y, 0, Y)$.

Recall that (quadratic) sprays correspond to linear connections. In terms of the horizontal bundle $\mathscr{H}$, linearity is expressed as $\mathscr{H}_{a v}=a_{*} \mathscr{H}_{v}$ for $a \in \mathbb{R}$ considered as a map $T M \rightarrow T M$ and for $v \in T M$. Thus one has

$$
\mathscr{H}_{a v}=a_{*} a^{m-1} \mathscr{H}_{v}
$$

as the second defining equation, together with (4.1), of a connection that is $h(m)$.

Note that for an $h(m)$ semispray $S$ with integral $m$, Grifone's [18] associated (generalized) Christoffel symbols $\Gamma$ are $h(m-1)$, appropriately. See (4.6) below for our version, which allows for nonlinear, including inhomogeneous, connections.

Here is the SODE induced by a connection. We shall call it the geodesic quasispray associated to the connection and its geodesics the geodesics of the connection. 
Theorem 4.1 For each connection $\mathscr{H}$, there is an induced SODE $S$ given by

$$
S(v)=\left.\pi_{*}\right|_{\mathscr{H}_{v}} ^{-1}(v)
$$

where $\pi: T M \rightarrow M$ is the natural projection and $v \in T M$. We write $\mathscr{H} \vdash S$ to denote this relationship. It is clear that this SODE is horizontal, so compatible with the given connection.

This proof and many of those that would follow are straightforward modifications of those found in [23] and we omit them here; more details are in [13].

Unfortunately, when the connection is $h(m-1)$ this SODE is not homogeneous as a SODE; it is only an $h(m)$ vector field on $T M$. In order to avoid this problem, we must consider a new type of (partial) homogeneity for connections.

Definition 4.2 A connection $\mathscr{H}$ on $T M$ is vertically homogeneous of degree $m$, denoted by $v h(m)$, if and only if

$$
\mathscr{H}_{a v}=a_{*} a_{v}^{m-1} \mathscr{H}_{v}
$$

where $a_{V}^{n}$ denotes scalar multiplication by $a^{n}$ in the vertical bundle.

More explicitly, $a_{*} a_{V}^{m-1}(x, y, X, Y)=\left(x, a y, a X, a^{m} Y\right)$ in local coordinates. Note that $h(m)$ and $v h(m)$ coincide only for $m=1$, the linear connections.

Proposition 4.3 If $\mathscr{H}$ is a connection with geodesic quasispray $S$, then $S$ is $h(m)$ if and only if $\mathscr{H}$ is $v h(m-1)$.

Connections may also be seen as sections of the bundle $G_{H}(T T M)$ of all possible horizontal spaces, a subbundle of the Grassmannian bundle $G_{n}(T T M)$. It is easily seen [13] that $G_{H}(T T M)$ is an affine bundle (bundle of affine spaces, vs. vector spaces). Thus a connection, being a section of this bundle, provides a choice of distinguished point in each fiber, hence a vector bundle structure on this affine bundle. If we consider only those connections compatible with a given SODE $S$, we obtain an affine subbundle $G_{H}^{S}(T T M)$ of $G_{H}(T T M)$, with fibers being pencils of possible horizontal spaces.

Theorem 4.4 Given a SODE $S$ on $M$, there exists a compatible connection $\mathscr{H}$ in $T T M$. 
Since the fibers of $G_{H}^{S}(T T M)$ are contractible, this is an easy exercise in obstruction theory [14, Ch. 8]; however, an explicit construction is desirable. It provides a concrete representation for our generalization of the AmbrosePalais-Singer (APS) correspondence.

Proof: Let $\Phi$ denote the local flow of $S$ and $\gamma$ an integral curve of $S$ with $\gamma(0)=v \in T_{p} M$. The basic idea is to use $S$ and $\Phi$ to define notions of horizontal and parallel which will coincide with the usual ones along $\gamma$ for any $\mathscr{H} \vdash S$. This is essentially the same as the usual construction [23]. The problem is that for inhomogeneous $S$, the ray $\{t v\}$ in $T_{p} M$ does not exponentiate to a geodesic in $M$.

To remedy this, we proceed as follows. For each $v \in T_{p} M$, choose $\varepsilon_{v}$ so that $\exp _{p}^{\varepsilon_{v}} v$ is defined. Such $\varepsilon_{v}$ exist by Proposition 3.1. For $0 \leq t \leq \varepsilon_{v}$, define

$$
\alpha_{v}(t)=\left(\exp _{p}^{\varepsilon_{v}}\right)^{-1} \exp _{p}^{t} v .
$$

Then $\alpha_{v}(0)=0, \alpha_{v}\left(\varepsilon_{v}\right)=v \in T_{p} M$, and $\alpha_{v}$ exponentiates to the geodesic with initial condition $v$ at $p$. Note that if $S$ is homogeneous, then $\alpha_{v}(t)=t v$.

We have a vector bundle map $\mathcal{J}: \pi^{*} T M \rightarrow \mathscr{V}$ which is an isomorphism on fibers. It is one version of canonical parallel translation on a vector space, identifying the tangent space at each point with the vector space itself. Now, for each $w \in T_{p} M$ define

$$
\mathscr{H}_{w}=\left\{\left.\frac{d}{d t}\right|_{t=0} \pi_{*} \Phi_{t *} \partial_{\alpha_{v}(t)} w \mid v \in T_{p} M\right\} .
$$

Clearly, this does not depend on the choices of $\varepsilon_{v}$ made earlier. (Note we are evaluating at 0 .) If $S$ is quadratic, it is easy to check that this coincides with the usual construction as found in [23, pp.96-97], since in that case $\exp _{p} t v=\pi \Phi(t, v)$ for $v \in T_{p} M$. The proof that $\mathscr{H}$ so defined is a connection and that $\mathscr{H} \vdash S$ follows Poor's proof of 2.98 [23, pp. 97-99] mutatis mutandis.

These connections will be our "standard" - our generalization of torsion-free linear connections.

We further note that admissible atlases correspond to certain reductions of the structure group of $T T M$ from $G L_{2 n}$ to $G L_{n} \oplus G L_{n}$, those which in turn correspond to direct-sum decompositions of $T T M$ in which one of the summands is the vertical bundle $\mathscr{V}$ (and the other is perforce a horizontal bundle), hence to connections in TTM. Thus any homogeneous SODE $S$ 
comes with a particular associated compatible connection $\mathscr{H}$, the one corresponding to the associated admissible atlas; $c f$. after Definition 2.2. Note, however, that it may not be the one naturally associated by the preceding construction.

There is another natural vector bundle map $K: \mathscr{V} \rightarrow T M$ respecting $\pi_{T}$ which is an isomorphism on fibers, another version of canonical parallel translation of a vector space. Using this, we define a connection map or connector for an arbitrary connection and thence a covariant derivative. Here $H$ is the horizontal projection, so $\mathscr{H}=\operatorname{im} H$ is the horizontal bundle.

Definition 4.5 For a connection $\mathscr{H}$, define the associated connector $\kappa$ : $T T M \rightarrow T M: z \mapsto K\left(z-H_{v} z\right)$ for $z \in T_{v} T M$.

Proposition 4.6 The connector $\kappa$ is a vector bundle map respecting $\pi_{T}$ but not $\pi_{*}$ in general. It respects $\pi_{*}$ if and only if the connection is linear.

According to Besse [7, p. 32f], a symmetric connector (connection) is invariant under the natural involution $J$ of $T T M$. Clearly this is possible only for linear connections.

Definition 4.7 The covariant derivative associated to the connection $\mathscr{H}$ is the operator defined by

$$
\nabla_{u} v=\kappa\left(v_{*} u\right)=K\left(v_{*} u-H_{v} v_{*} u\right)
$$

and is tensorial in $u$ but nonlinear (in general) in $v$.

This last comes from the general lack of respect for the $\pi_{*}$ structure by $\mathscr{H}$, $H$, and $\kappa$.

Example 4.8 We always have $\nabla_{0} v=0$. For any $v h(m)$ connection, $\nabla_{u} a v=$ $K\left(a_{*} v_{*} u-H_{a v} a_{*} v_{*} u\right)=a K\left(v_{*} u-a_{V}^{m-1} H_{v} v_{*} u\right)$, and similarly for homogeneous ones. So (vertically) homogeneous connections do not differ significantly from linear ones. In particular, $\nabla_{u} 0=0$ for all $u$ for all (vertically) homogeneous connections; in fact, they all have the same horizontal spaces along the 0 -section of $T M$, namely the subspaces tangent to it (i.e., those in the image of $\left.0_{*}: T M \rightarrow T T M\right)$. We call all such connections sharing this property 0-preserving; they differ minimally from (vertically) homogeneous (including linear) connections. In contrast, connections with $\nabla_{u} 0 \neq 0$ for even some $u$ are much farther from linear; we call them strongly nonlinear. 
Theorem 4.9 There is a bijective correspondence between (possibly nonlinear) connections $\mathscr{H}$ and our (possibly nonlinear) covariant derivatives $\nabla$ on $T M$.

The proof is a relatively straightforward modification of $[23$, p. 77 , proof of 2.58]; see [13] for details. Thus as usual, we may refer indifferently to $\mathscr{H}$ or its associated $\nabla$ as the connection.

Generalized Christoffel symbols may be introduced through

$$
\left(K H_{v} v_{*} u\right)^{k}=\Gamma_{i}^{k}(v) u^{i},
$$

making manifest the tensoriality in $u$. Here are some examples of their use. Observe that $\left(K v_{*} u\right)^{k}=u^{i} \partial_{i} v^{k}$ so that

$$
\left(\nabla_{u} v\right)^{k}=u^{i} \partial_{i} v^{k}-\Gamma_{i}^{k}(v) u^{i}
$$

is the covariant derivative. The geodesic equation is

$$
\ddot{c}^{k}=\Gamma_{i}^{k}(\dot{c}) \dot{c}^{i}
$$

which means that

$$
\mathcal{S}^{k}(\dot{c})=\Gamma_{i}^{k}(\dot{c}) \dot{c}^{i}
$$

for $S$ the quasispray induced by the connection $\nabla$. Note that we can write any $\mathcal{S}^{k}(v)$ in the form

$$
\mathcal{S}^{k}(v)=\Gamma_{i}^{k}(v) v^{i},
$$

although $\Gamma$ may be less well-behaved than $\mathcal{S}$. In this way we can obtain the standard ("torsion-free") connection associated to $S$ by our generalized APS construction (proof of Theorem 4.4).

We obtain the usual relation between two notions of geodesic.

Theorem 4.10 A curve $c$ is a geodesic of $\mathscr{H}$ if and only if $\nabla_{\dot{c}} \dot{c}=0$.

Proof: $\nabla_{\dot{c}} \dot{c}=\kappa\left(\dot{c}_{*} \dot{c}\right)=K\left(\dot{c}_{*} \dot{c}-H_{\dot{c}} \dot{c}_{*} \dot{c}\right)=K\left(\dot{c}_{*} \dot{c}-S(\dot{c})\right)$ by the construction of $S$ in Proposition 4.1. Now all we have to do is identify $\dot{c}_{*} \dot{c}$ as $\ddot{c}$ and recall that $K$ is an isomorphism on fibers.

Curvature is readily handled. Let $\mathscr{H}$ be a connection on $M$. The horizontal lift of a vector field $U$ on $M$ is denoted by $\bar{U}$. 
Definition 4.11 Given vector fields $U$ and $V$ on $M$, the curvature operator $R(U, V): T M \rightarrow T M$ is defined by

$$
R(U, V) w=\kappa\left([\bar{V}, \bar{U}]_{w}\right)
$$

for all $w \in T M$. It is tensorial in the first two arguments, but nonlinear (in general) in the third.

The arguments are reversed on the right in order to obtain the usual formula in terms of the associated covariant derivative,

$$
R(U, V) W=\nabla_{U} \nabla_{V} W-\nabla_{V} \nabla_{U} W-\nabla_{[U, V]} W,
$$

as one may verify readily. It is also easy to check that this curvature vanishes if and only if $\mathscr{H}$ is integrable, thus justifying our definition.

Torsion is considerably more obscure. Essentially, what we do is to regard the usual torsion formula as derived from the difference operator (difference tensor in the linear case) construction; $c f$. [23, pp. 99-100]. Then we make the notion of torsion-free connection fundamental and determine what torsion has to be in order to make it consistent with the classical linear case; see [13] for complete details.

\section{References}

[1] M. Abate and G. Patrizio, The homogeneous complex Monge-Ampère equation and Finsler metrics, in Seminars of geometry, University of Bologna, Italy, 1994-1995, ed. S. Coen. Bologna: Univ. degli Studi di Bologna, Dip. di Matematica, 1996. pp.1-25.

[2] W. Ambrose, R. S. Palais and I. M. Singer, Sprays, Anais Acad. Brasil Ciênc. 32 (1960) 163-178.

[3] P.L. Antonelli and M. Anastaseie, The Differential Geometry of Lagrangians which Generate Sprays. Fund. Theor. Phys. 76. Dordrecht: Kluwer, 1996.

[4] P. L. Antonelli, R. S. Ingarden and M.S. Matsumoto, The Theory of Sprays and Finsler Spaces with Applications in Physics and Biology. Fund. Theor. Phys. 58. Dordrecht: Kluwer, 1993.

[5] G. S. Asanov, Finsler Geometry, Relativity and Gauge Theories. Dordrecht: Kluwer, 1985. 
[6] D. Bao, S.-S. Chern, and Z. Shen, An Introduction to Riemann-Finsler Geometry. New York: Springer, 2000.

[7] A. L. Besse, Manifolds all of Whose Geodesics are Closed. New York: Springer-Verlag, 1978.

[8] Misha Bialy and Leonid Polterovich, Geodesics of Hofer's metric on the group of Hamiltonian diffeomorphims, Duke Math J. 76 (1994) 273-292.

[9] F. Brickell and R.S. Clark, Differentiable Manifolds. New York: Van Nostrand, 1970.

[10] Th. Bröcker and K. Jänich, Introduction to Differential Topology. Cambridge: U.P., 1982.

[11] L. Del Riego and P.E. Parker, Pseudoconvex and disprisoning homogeneous sprays, Geom. Dedicata 55 (1995) 211-220.

[12] L. Del Riego and P. E. Parker, Some nonlinear planar sprays, in Nonlinear Analysis in Geometry and Topology, ed. T. M. Rassias. Palm Harbor: Hadronic Press, 2000. pp. 21-52.

[13] L. Del Riego and P. E. Parker, Generalized Sprays and Nonlinear Connections, DGS preprint DRP3, 22 pp. arXiv: math.DG/0304067

[14] C. T. J. Dodson and P. E. Parker, A User's Guide to Algebraic Topology. Boston: Kluwer Academic Publishers, 1997.

[15] P. Dombrowski, On the geometry of the tangent bundle, J. reine angew. Math. 210 (1962) 73-88.

[16] T. Duchamp and M. Kalka, Singular Monge-Ampère foliations, Math. Ann. 325 (2003) 187-209.

[17] J. Grifone, Connexions non linéaires conservatives, C. R. Acad. Sci. Paris Sér. A Math. 268 (1969) 43-45.

[18] J. Grifone, Structure Presque Tangent et Connexions non Homogènes. Thèse $3^{\text {ème }}$ cycle, Université de Grenoble, 1971.

[19] M. W. Hirsch and S. Smale, Differential Equations, Dynamical Systems, and Linear Algebra. New York: Academic Press, 1974. 
[20] J. Klein and A. Voutier, Formes extérieures géneratrices de sprays, Ann. Inst. Fourier 18 (1968) 241-260.

[21] M. de León and P. Rodríguez, Methods of Differential Geometry in Analytical Mechanics. Amsterdam: North-Holland, 1989.

[22] Leonid Polterovich, Geometry on the group of Hamiltonian diffeomorphisms, Doc. Math. J. Extra Volume ICM II (1998) 401-410.

[23] W.A. Poor, Differential Geometric Structures. New York: McGrawHill, 1981.

[24] H. Reckziegel, Generalized sprays and the theorem of Ambrose-PalaisSinger, in Geometry and Topology of Submanifolds V, ed. F. Dillen, L. Vrancken, L. Verstraelen, and I. Van de Woestijne. River Edge: World Scientific, 1993. pp. 242-248.

[25] A. Vondra, Sprays and Homogeneous Connections on $\mathbb{R} \times T M$, Arch. Math. (Brno) 28 (1992) 163-173. 